\title{
Unhealthy Behaviours: An International Comparison
}

\author{
Fabrizio Ferretti* \\ Department of Communication and Economics, School of Social Sciences, University of Modena and Reggio \\ Emilia, Reggio Emilia, Italy \\ * fabrizio.ferretti@unimore.it
}

\section{Abstract}

In the current global economy, chronic non-communicable diseases (NCDs) have become the leading cause of death and a major health concern for both developed and developing countries. Among other factors, the worldwide spread of NCDs is driven by the globalisation of unhealthy habits. The purpose of this paper is to develop a simple statistic to measure, at the national level, the average population's exposure to the main NCDs modifiable risk factors. The approach and methodology followed by the United Nations Development Programme to compute the Human Development Index $(H D I)$ is applied to four basic indicators of NCD-related preventable risk factors (alcohol consumption, excess caloric intake, nonbalanced diet and tobacco use) in 112 countries worldwide in 2012-14. We obtain a summary composite index, which we call the Unhealthy Behaviour Index (UBI), which ranks countries by the average level of the unhealthy habits (drinking, eating and smoking) of their populations. We find that Belarus and Russian federation are the two countries with the unhealthiest NCD-related lifestyle. With the exception of Canada, the first twenty populations more exposed to the main NCDs preventable risk factors all live in European countries, and mainly in countries of Eastern Europe. Overall, the UBI tends to increase along with the level of human development. In medium, high and very high $H D I$ countries, however, the same level of human development may be associated with very different kinds of NCD-related lifestyles. Finally, economic growth may push populations toward either more unhealthy or healthy habits, depending on the countries' level of development; the elasticity of unhealthy habits with respect to income per capita is positive (but less than one: on average 0.6) until $\$ 30,000$, decreases as income rises, and becomes negative (around -0.3 ) in very high income countries.

Copyright: @ 2015 Fabrizio Ferretti. This is an open access article distributed under the terms of the Creative Commons Attribution License, which permits unrestricted use, distribution, and reproduction in any medium, provided the original author and source are credited.

Data Availability Statement: All relevant data are within the paper and its Supporting Information files.

Funding: The authors have no support or funding to report.

Competing Interests: The authors have declared that no competing interests exist.

\section{Introduction}

Once considered 'diseases of affluence' that affected mostly elderly and wealthy people in Western advanced countries, today, non-communicable diseases (NCDs) have become one of the major global health concerns, as well as the leading cause of death worldwide [1]. According to the latest World Health Organization (WHO) global report, NCDs: “. . . were responsible for 38 
million (68\%) of the world's 56 million deaths in 2012. More than $40 \%$ of them (16 million) were premature deaths under the age of 70 years. Almost three-quarters of all NCD deaths (28 million) and the majority of premature deaths ( $82 \%)$ occur in low- and middle-income countries" ([2], p. XI).

The increasing worldwide burden of NCDs is the result of complicated interactions between several demographic, economic and social structural changes [3], and is strongly associated with the globalisation of unhealthy lifestyles [4-6]. The leading four chronic NCDs-that is, cardiovascular diseases, chronic respiratory diseases, cancers, and diabetes [7] - are characterised by a complex aetiology, but generally stem from a combination of non-modifiable risk factors (e.g., sex, age and the inborn genetic characteristics of individuals), and a well-known set of modifiable risk factors: primarily, tobacco use, alcohol abuse, qualitative and quantitative unhealthy nutrition, lack of physical activity, environmental pollution and chronic infection [8]. This is why epidemiological studies emphasise the role of primary prevention to tackle NCDs and suggest effective ways to drastically reduce the global incidence of NCDs by controlling the main lifestyle-related risk factors in each country $[9,10]$.

Recent developments in NCD epidemiology highlight the importance of measuring the combined effects of multiple lifestyle risk behaviours on people's health outcomes [11]. To date, the research has helped to understand and explain this phenomenon at the individual (i.e., microeconomic) level [12]. The purpose of this paper is to develop a simple statistic in order to measure the average population's exposure to the main NCD modifiable risk factors at the country (i.e., macroeconomic) level. In what follows, the approach and methodology followed by the United Nations Development Programme (UNDP) to compute the HDI (Human Development Index) [13] is applied to four of the main preventable risk factors that underlie the leading NCDs (alcohol abuse, excess caloric intake, non-balanced diet, and tobacco use) in 112 countries worldwide for the period 2012-14. The result is a summary composite indexthat will be called the 'Unhealthy Behaviour Index (UBI)' - which allows us to rank countries by the average level of the unhealthy (drinking, eating and smoking) habits of their respective populations.

\section{Methods and Data}

It is often useful to construct a composite indicator to summarise a wide range of indicators of a multi-dimensional phenomenon in a single statistic [14]. In this paper, we construct the UBI, a summary measure of three fundamental dimensions of people's health-related habits and behaviours: drinking, eating and smoking (Fig 1). These key lifestyle factors are captured here by four basic indicators of the main NCD modifiable risk factors [15]: i.e. alcohol consumption $(A L C)$, excess caloric intake (ECI), non-balanced diet (that is, a diet too rich in total fat and protein, $N B D$ ) and tobacco use $(T O B)$.

Each indicator is transformed into a corresponding normalised index $\left(I_{x}\right)$ according to the standard HDI methodology [16] as follows:

$$
I_{x}=\frac{a c t_{x}-\min _{x}}{\max _{x}-\min _{x}}
$$

where $a c t_{x}, \min _{x}$ and $\max _{x}$ stand for the actual, minimum and maximum value of the underlying indicator. For the eating dimension, Eq 1 is applied to each of the two subcomponents (ECI and $N B D$ ). Then, a geometric mean of the resulting sub-indices (ECIX and NBDX) is created, and Eq 1 is applied again to the geometric mean of these two indices in order to obtain the nutrition dimension index (NUTX). Finally, $U B I$ is computed as the geometric mean of the three normalised dimension indices-that is, alcohol (ALCX), nutrition (NUTX), and tobacco 
DIMENSIONS

INDICATORS

consumption

ALC

Drinking

Alcohol
consumption
ALC

SUBINDICES

DIMENSION INDEX

Alcohol index

ALCX
Eating

Excess
caloric intake

ECI
Non-

balanced diet

NBD
Smoking

Tobacco

use

TOB

$\downarrow$

Unhealthy Behaviour Index (UBI)

Fig 1. The Unhealthy Behaviour Index (UBI): Graphical presentation.

doi:10.1371/journal.pone.0141834.g001

$(T O B X)$-as follows:

$$
U B I=\left(A L C X^{1 / 3} \times N U T X^{1 / 3} \times T O B X^{1 / 3}\right)
$$

Computing Eq 2 requires data on several variables (Table 1). Specifically, $A L C$ is measured by the per capita consumption of pure alcohol (litres/person15+/year) estimated for 2012 by the WHO $[2,17]$. TOB is measured by the per capita consumption of cigarettes (number/person/year) estimated for 2012 by Ng et al. [18]. ECI and NBD are measured by the per capita intake of energy (kcal/day) and of total fat and protein ( $\mathrm{g} /$ day) over the recommended level,

Table 1. Variables and goalposts for the Unhealthy Behaviour Index.

\begin{tabular}{|c|c|c|c|c|c|}
\hline Variable & Code & Description & Source & Obs. max. & Min. \\
\hline $\begin{array}{l}\text { Alcohol } \\
\text { consumption }\end{array}$ & $A L C$ & $\begin{array}{l}\text { Per capita consumption of pure alcohol (litres/person } 15 \\
\text { +/year) }^{\mathrm{a}}\end{array}$ & WHO (2014) & 17.8 (Belarus, 2012) & 0 \\
\hline $\begin{array}{l}\text { Excess caloric } \\
\text { intake }\end{array}$ & $\mathrm{ECl}$ & $\begin{array}{l}\text { Actual-Recommended caloric intake ECI = DES-ADER } \\
\left(\mathrm{kcal} / \text { person/day) }{ }^{\mathrm{c}}\right.\end{array}$ & FAO (2014) & $\begin{array}{l}\text { 1,349.5 (Turkey, 2012- } \\
2014)^{\mathrm{b}}\end{array}$ & 0 \\
\hline Non-balanced diet & $N B D$ & $\begin{array}{l}\text { Actual-Recommended fat and protein intake NBD = AFPS- } \\
\text { RFPI (g/person/day) }\end{array}$ & $\begin{array}{l}\mathrm{FAO}(2014) \text { and } \mathrm{FAO} / \\
\mathrm{WHO}(2003)\end{array}$ & $\begin{array}{l}145.1 \text { (Israel, 2012- } \\
2014)\end{array}$ & 0 \\
\hline Tobacco use & TOB & Per capita cigarettes consumption (number/person/year) & $\mathrm{Ng}$ et al. (2012) & 3,385 (Cyprus, 2012) & 0 \\
\hline
\end{tabular}

$\mathrm{a}_{15+}=$ person aged 15 or older.

b2012-2014 = three years average.

${ }^{\mathrm{C} D E S}=$ Dietary Energy Supply; ADER = Average Dietary Energy Requirement.

${ }^{\mathrm{d}} \mathrm{AFPS}=$ Average fat and protein supply; RFPI = Recommended fat and protein intake.

doi:10.1371/journal.pone.0141834.t001 
estimated for 2012-14 (three-year average) by the United Nations Food and Agricultural Organization (FAO) and the WHO $[19,20]$.

About the two NUTX sub-indices, ECI is calculated from the FAO database [19] as the difference between the Dietary Energy Supply $(D E S)$ and the Average Dietary Energy Requirement ( $A D E R$ ), whereas $N B D$ is calculated, again, from the FAO database as the difference between the actual and the recommended total fat and protein intake (AFPS and RFPI, respectively). Intake recommendations are taken from the joint $\mathrm{FAO}-\mathrm{WHO}$ intake goals to prevent diet- and nutrition-related chronic NCDs [20] (see S1 Appendix for details on these calculations). $E C I$ and $N B D$ are, therefore, basic indicators of a population's incorrect eating habits, characterised by the excessive and unhealthy consumption of calorie-, fat-, and protein-dense foods.

Minimum and maximum values (or goalposts) for each indicator are set in order to transform variables into a corresponding index between 0 and 1 . The main purpose of the $U B I$ is to provide a country summary measure of multiple health risk behaviours, in which populations with the most NCD-related unhealthy habits receive the highest UBI scores. To this end, maximum values are simply the highest observed values in the sample-i.e. ALC, 17.8 litres (Belarus), $E C I, 1,349.5 \mathrm{kcal}$ (Turkey), NBD, $145.1 \mathrm{~g}$ (Israel), and TOB, 3,385 cigarettes (Cyprus), respectively. Minimum values are set equal zero for all indicators.

The choice to set $\min _{x}$ equal to zero is straightforward for tobacco use, as the optimal level of cigarette consumption is always equal to zero. In a similar way, since positive values of both $E C I$ and $N B D$ measure excess dietary deviations from the international standard guidelines to prevent NCDs, it is reasonable to set the minimum deviation from the correct energy, total fat, and protein intake equal to zero. Finally, despite moderate alcohol consumption seeming to provide mild protection against selected NCDs [21], the minimum value of $A L C$ is also set equal to zero, because several studies suggest that this moderate level is very low [22-24], and is associated not with alcohol itself, but to the polyphenols contained only in select alcoholic beverages (especially red wine) [25].

Combining the WHO [2,17], Ng et al. [18] and FAO [19] databases, there are 159 countries with no missing data for the four indicators ( $A L C, E C I, N B D$ and TOB). However, 47 countries -which have a history of issues of undernourishment-have negative values for either or both $E C I$ and NBD. Since our focus is on people's unhealthy lifestyle choices, we will compute the index only within the subset of the 112 remaining countries, where both nutrition indicators assume positive values (see Tables B and C in S1 File for the full and the reduced database).

Let us compute, as an example, the UBI in the United Kingdom (Table 2). In 2012, the estimated UK consumption of alcohol and cigarettes per capita was 11.4 litres/year and 998

Table 2. Calculating the Unhealthy Behaviour Index (example: United Kingdom).

\begin{tabular}{llclllr}
\hline Variable & Code & United Kingdom & Max & Min & Subindices & Dimension index \\
\hline Alcohol consumption & $A L C$ & 11.4 & 17.8 & 0 & $A L C X$ & $\mathbf{0 . 6 4 0}$ \\
Excess caloric intake & $E C I$ & 922.3 & $1,349.5$ & 0 & $E C I X$ & 0.683 \\
Non balanced diet & $N B D$ & 106.8 & 145.1 & 0 & NBDX & 0.736 \\
& & & & & gm $(E C I X, N B D X)^{\mathrm{a}}$ & 0.709 \\
Tobacco consumption & TOB & 998.0 & 3,385 & 0 & TOBX & $\mathbf{0 . 7 3 4}$ \\
& & & & & Unhealthy Behaviour Index-UBI & $\mathbf{0 . 2 9 5}$ \\
\hline
\end{tabular}

${ }^{\mathrm{a}} \mathrm{gm}(\mathrm{ECIX}, \mathrm{NBDX})=$ geometric mean of ECIX and NBDX.

doi:10.1371/journal.pone.0141834.t002 
cigarettes/year, respectively. Furthermore, from 2012-14, the UK per capita intake of calories and of total fat and protein were estimated well above the requirement of the population to prevent NCDs (specifically, an excess of $922.3 \mathrm{kcal} /$ day and $106.8 \mathrm{~g} /$ day, respectively). Given the goalposts, according to Eq 1, these figures give:

$$
\begin{gathered}
A L C X_{U K}=\frac{11.4-0}{17.8-0}=0.640 \\
\operatorname{TOBX}_{U K}=\frac{998-0}{3,385.0-0}=0.295 \\
E_{U K}=\frac{922.3-0}{1,349.5-0}=0.683 \\
\operatorname{NBDX}_{U K}=\frac{106.8-0}{145.1-0}=0.736
\end{gathered}
$$

Therefore, computing the geometric mean of $E C I X_{U K}$ and $N B D X_{U K}$, and reapplying Eq 1 to the result, gives:

$$
\operatorname{NUTX}_{U K}=\frac{\sqrt{0.683 \times 0.736}-0}{0.967-0}=0.734
$$

This value entered into Eq 2, along with $A L C X_{U K}$ and $T O B X_{U K}$ gives:

$$
U B I_{U K}=\sqrt[3]{0.640 \times 0.734 \times 0.295}=0.517
$$

Equation shows a UBI value for the UK's population of around 0.52 , in which a diet too rich in both fat and protein, an excess of caloric intake, and a non-negligible level of alcohol consumption are partially offset by a relatively modest tobacco use.

\section{Results}

The results of applying the above methodology to the set of 112 countries worldwide are summarised in Table 3, where the first 20 countries for the unhealthy habits of their populations are listed, as measured by the UBI and by its three dimension indices (see Tables C and D in S1 File for the full results and the full country ranking).

Belarus is the population with the highest $U B I$ value in the sample (0.77), and thus, with the worst exposure to NCD lifestyle risk factors, strictly followed by the populations of the Russian Federation and Greece ( 0.69 and 0.68 , respectively). It is worthwhile to note that, with the exception of Canada, the first column of Table 3 contains only European countries: mainly countries from Eastern Europe. This evidence is even more striking for the drinking dimension, where Australia is the only non-European country, and the first top ten positions are all occupied by Eastern European populations. In the same way, an NCD prone lifestyle for tobacco use tends to prevail in countries in Eastern Europe (especially from Balkans) and from the Mediterranean region (i.e., Cyprus, Greece, and Malta). Finally, the composition of the first ten populations for unhealthy habits changes partially for the eating dimension. Israel has the highest score, and Eastern European countries are replaced by European Union (Austria, Belgium, France, Luxemburg, Italy and Ireland) and North American (the USA and Canada) countries.

Overall, unhealthy lifestyles tend to increase with the level of human development, as measured by the HDI. This is shown in Fig 2, which plots the UBI against the HDI for each country 
Table 3. Ranking of the first ten countries by $U B I$ and dimension indices.

\begin{tabular}{|c|c|c|c|c|c|c|c|c|}
\hline & Country & UBI & Country & $A L C X$ & Country & NUTX & Country & ТОВХ \\
\hline 1 & Belarus & 0.773 & Belarus & 1.000 & Israel & 1.000 & Cyprus & 1.000 \\
\hline 2 & Russian Fed. & 0.694 & Lithuania & 0.949 & Austria & 0.954 & Malta & 0.961 \\
\hline 3 & Greece & 0.687 & Moldova & 0.904 & United States & 0.919 & Belarus & 0.856 \\
\hline 4 & Czech Rep. & 0.668 & Russian Fed. & 0.831 & Belgium & 0.917 & Russian Fed. & 0.838 \\
\hline 5 & Ireland & 0.651 & Czech Rep. & 0.787 & France & 0.874 & Croatia & 0.819 \\
\hline 6 & Montenegro & 0.647 & Ukraine & 0.787 & Canada & 0.837 & Moldova & 0.816 \\
\hline 7 & Austria & 0.644 & Romania & 0.742 & Luxembourg & 0.827 & Lebanon & 0.804 \\
\hline 8 & France & 0.634 & Croatia & 0.730 & Montenegro & 0.822 & Greece & 0.803 \\
\hline 9 & Romania & 0.633 & Slovakia & 0.702 & Italy & 0.821 & St. Vincent ${ }^{\mathrm{a}}$ & 0.795 \\
\hline 10 & Croatia & 0.630 & Hungary & 0.697 & Ireland & 0.800 & Bosnia-Herz. & 0.788 \\
\hline 11 & Lithuania & 0.626 & France & 0.691 & Greece & 0.780 & Macedonia & 0.748 \\
\hline 12 & Malta & 0.624 & Serbia & 0.691 & Norway & 0.776 & Slovakia & 0.716 \\
\hline 13 & Luxembourg & 0.615 & Portugal & 0.685 & Iceland & 0.775 & Montenegro & 0.673 \\
\hline 14 & Belgium & 0.606 & Latvia & 0.674 & Turkey & 0.772 & Serbia & 0.672 \\
\hline 15 & Switzerland & 0.599 & Australia & 0.669 & Germany & 0.751 & Armenia & 0.664 \\
\hline 16 & Canada & 0.590 & Luxembourg & 0.669 & Portugal & 0.746 & Estonia & 0.661 \\
\hline 17 & Poland & 0.589 & Finland & 0.657 & Switzerland & 0.738 & Korea Rep. & 0.646 \\
\hline 18 & Latvia & 0.584 & Poland & 0.652 & United Kingdom & 0.734 & Czech Rep. & 0.625 \\
\hline 19 & Slovenia & 0.579 & Germany & 0.646 & Australia & 0.702 & Uruguay & 0.610 \\
\hline 20 & Kazakhstan & 0.574 & United Kingdom & 0.640 & Lithuania & 0.695 & Ireland & 0.608 \\
\hline
\end{tabular}

a Saint Vincent and the Grenadines

doi:10.1371/journal.pone.0141834.t003

in the sample. There is a clear positive relationship between the average levels of human development and unhealthy habits. However, by classifying countries according to their HDI into the four main UNDP's groups (i.e., very high, high, medium and low HDI) [26], it is interesting to note that, in medium, and especially in high and very high HDI countries, the relationship between $U B I$ and $H D I$ tends to become nearly vertical. In other words, the same level of human development may be associated with very different kinds of NCD-related lifestyles.

A fundamental component of the $H D I$ is the income per capita $(Y)$, usually measured by the Gross National Income per person (GNIPc, in PPP \$ [26]). Table 4 shows the average value of the $U B I$ and its components when countries are classified according to their per capita income level. On average, the UBI more than triples in 'rich' countries (i.e., with a GNIpc greater than $\$ 30,000$ ) with respect to the poorest one (where the GNIpc is less than $\$ 5,000$ ): 0.48 and 0.15 , respectively. However, the upper-middle (i.e., with a GNIpc between $\$ 15,001$ and $\$ 30,000$ ) and high-income countries show around the same unhealthy outcome, with better performance of the latter for the drinking and, especially, smoking dimensions. On the contrary, the excess of both energy and total fat-protein intake always increases from low to high income countries.

The relationship between economic development and unhealthy habits can be further examined by computing the elasticity of the $U B I$ with respect to income per capita-i.e. $\eta_{U Y}=$ $(d U B I / d Y) \times(Y / U B I)$ - which measures the percentage change of the $U B I$ when income changes by 1 percent. The result of the computation may help to understand and measure the impact of economic growth on health-related lifestyles. To do this, we regress GNIpc on UBI with a simple quadratic model $\left(U B I=\beta_{0}+\beta_{1} G N I p c+\beta_{2} G N I p c^{2}+\varepsilon\right)$ that, estimated using 


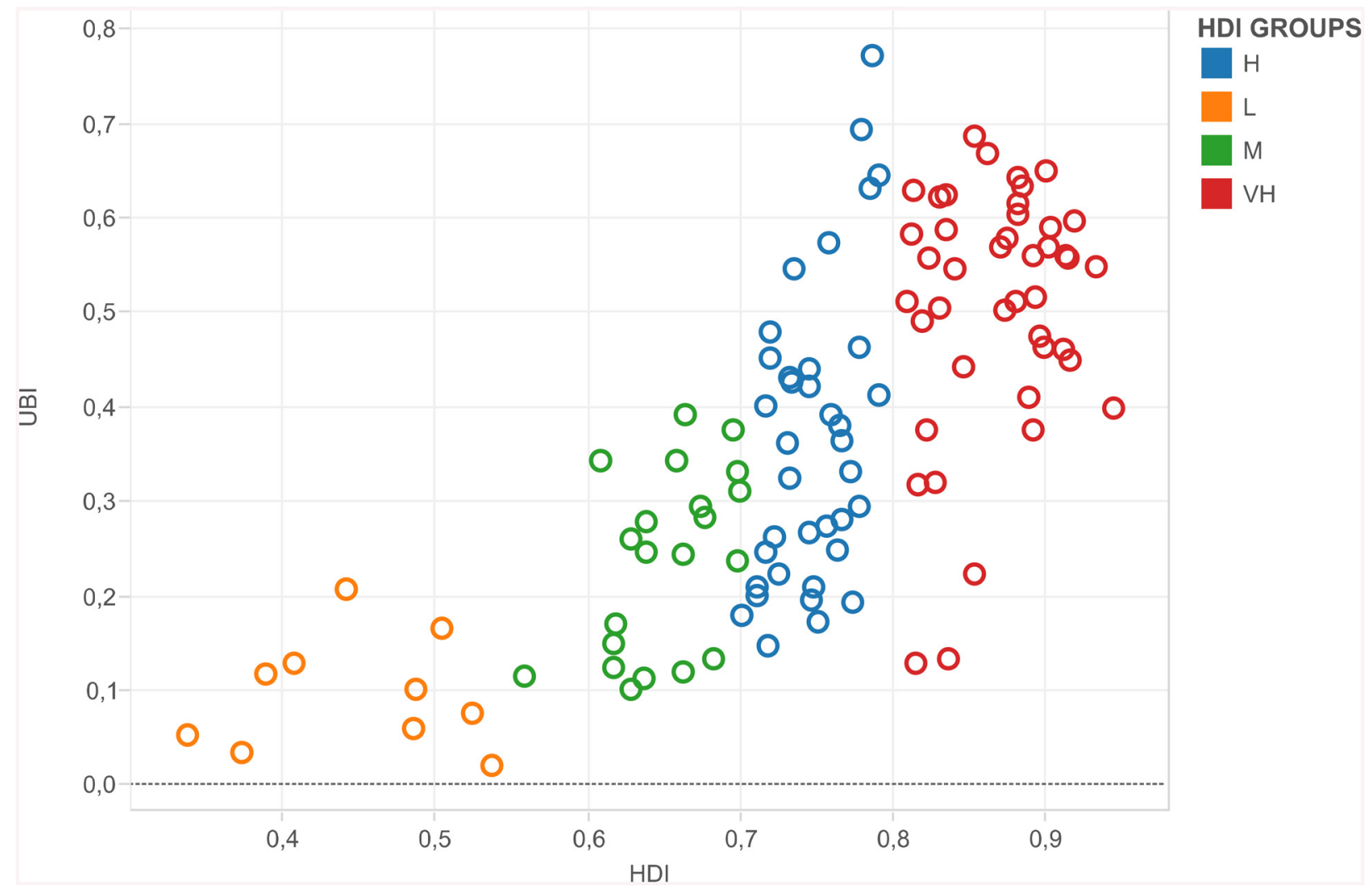

Fig 2. The Unhealthy Behaviour Index and the Human Development Index.

doi:10.1371/journal.pone.0141834.g002

White's coefficient covariance matrix to obtain heteroskedasticity robust standard errors, gives:

$$
\begin{array}{cc}
U B I=0.10+ & 2.25 \mathrm{E}-05 \text { GNIPc }-2.78 \mathrm{E}-10 \text { GNIp }^{2} \\
(1.88 \mathrm{E}-06) & (3.00 \mathrm{E}-11) \\
t=11.92 & -9.28
\end{array}
$$

$(n=112$, Adj. $R$-Sq. $=0.53)$. Therefore, $\eta_{U Y}$ is computed from Eq 9 as $\left(\beta_{1}+2 \beta_{2} G N I p c\right) \times$ (GNIpc/UBI), by using the average value of $U B I$ and GNIpc in each income group. The results, as shown in column 3 of Table 4, indicate that health-related habits are relatively inelastic to

\begin{tabular}{|c|c|c|c|c|c|c|c|}
\hline Income groups & $U B I$ & $n_{U Y}^{a}$ & $A L C$ & $E C I$ & NBD & ТОВ & $n^{\mathrm{b}}$ \\
\hline High $\left(\mathrm{GNI} p \mathrm{c}^{\mathrm{c}}>30.000\right)$ & 0.488 & -0.29 & 8.5 & 879.7 & 96.9 & 1,487 & 26 \\
\hline Upper middle (GNI pc 15.001-30.000) & 0.476 & 0.52 & 9.3 & 720.3 & 58.1 & 1,833 & 33 \\
\hline Lower middle (GNI pc 5.001-15.000) & 0.292 & 0.66 & 6.4 & 564.1 & 27.7 & 1,286 & 37 \\
\hline Low $(\mathrm{GNI} p c<$ or $=5.000)$ & 0.153 & 0.71 & 2.9 & 483.1 & 20.3 & 742 & 16 \\
\hline
\end{tabular}
income changes (all coefficients are less than one). But, it is noteworthy that $\eta_{U Y}$ is positive (on

Table 4. Unhealthy Behaviour Index and components by income groups.

${ }^{a} n_{U Y}=$ income elasticity of the UBI

$\mathrm{b}_{n}=$ number of countries of the sample in the income groups.

${ }^{c}$ GNI pc = Gross National Income per capita 2013 (2011, PPP \$) (UNDP, 2014), PPP is purchasing power parity.

doi:10.1371/journal.pone.0141834.t004 
average, 0.6 ) for populations with per capita income less than $\$ 30,000$, decreases from 0.7 to 0.5 as income rises from $\$ 5,000$ to $\$ 30,000$ per capita, and becomes negative (on average, - 0.3 ) in high income countries. In other words, sustained increases in income per capita may push populations toward more either unhealthy or healthy habits. However, both the direction and magnitude of these changes depend on the country's level of economic development. Finally, by setting the first derivative of Eq 9 equal to zero and solving for GNIpc, we can find an income turning point of about $\$ 40,500$. This is the threshold income level beyond which $\eta_{U Y}$ changes its sign, and economic growth starts exerting a positive effect on people's NCD-related lifestyles.

\section{Discussion}

Within a simple model of consumer behaviour, these empirical findings may help to better understand the channels through which economic growth and human development affect the incidence of NCDs in both advanced and emerging countries. Thus, in this section, we present an economic framework to ground our empirical work and discuss its implications for the epidemiology of NCDs.

In order to capture people's exposure to lifestyle risk factors, one may consider an NCDrelated consumption bundle in which each item is described by the list of its healthy and unhealthy characteristics. The whole set of these characteristics determines a more or a less risk prone lifestyle. To simplify, let us consider a representative market basket composed of healthy goods ( $h$, such as fruits and vegetables) and unhealthy goods ( $u$, such as high-fat and calorie-dense foods) [27].

Both kinds of goods yield immediate satisfaction, but exert opposite effects on future health outcomes. A main feature of NCD epidemiology is the delay between illness onset and the exposure to risk factors. That is, today's incidence rate is affected by yesterday's exposure, and today's exposure will affect tomorrow's incidence rate. Consuming $h$ and $u$ increases today's utility. However, utility from $u$ comes at a price of a greater exposure to NCD risk factors, and thus, of a greater likelihood to develop one or more NCDs in the future (and vice versa for $h$, whose consumption gives utility today and protects from NCDs, providing future health benefits) [28].

At any given time, the individual's preferences for $h$ and $u$ can be usefully described by an indifference map (as shown in Fig 3). Each indifference curve $\left(I_{1}, I_{2}, I_{3}\right.$ and $\left.I_{4}\right)$ represents all combinations of $h$ and $u$ that yields the same level of 'today's utility' (i.e., satisfaction in the current period). Because both more $h$ and $u$ make the individual better off today, indifference curves are downward sloping, and the further the curves are from the origin, the greater is the level of satisfaction that they represent [27].

At a low level of utility, however, indifference curves (such as $I_{1}$ ) are nearly right angles; that is, people are 'too poor' to be able to choose between $h$ and $u$. In these contexts, where a consumption bundle, such as $E$, is the one that is affordable to the majority of the population, analysing unhealthy behaviours is somewhat inappropriate. This is why the UBI is computed by deleting all the poorest countries from the sample. In other words, our analysis applies to societies with medium, high or very high levels of human development. Indeed, in each subset of these countries (as clearly shown in Fig 2), the same level of human development is associated with very different $U B I$ values. This means that, moving away from the origin in Fig 3, indifference curves become flatter (such as $I_{2}$ or $I_{3}$ ), and people may exert some degree of freedom on their health-related habits. At these corresponding level of utility, consumers are relatively free to choose between different consumption bundles (such as $F$ or $G$, on $I_{3}$ ), all of which represent a more healthy or unhealthy lifestyle, respectively. 


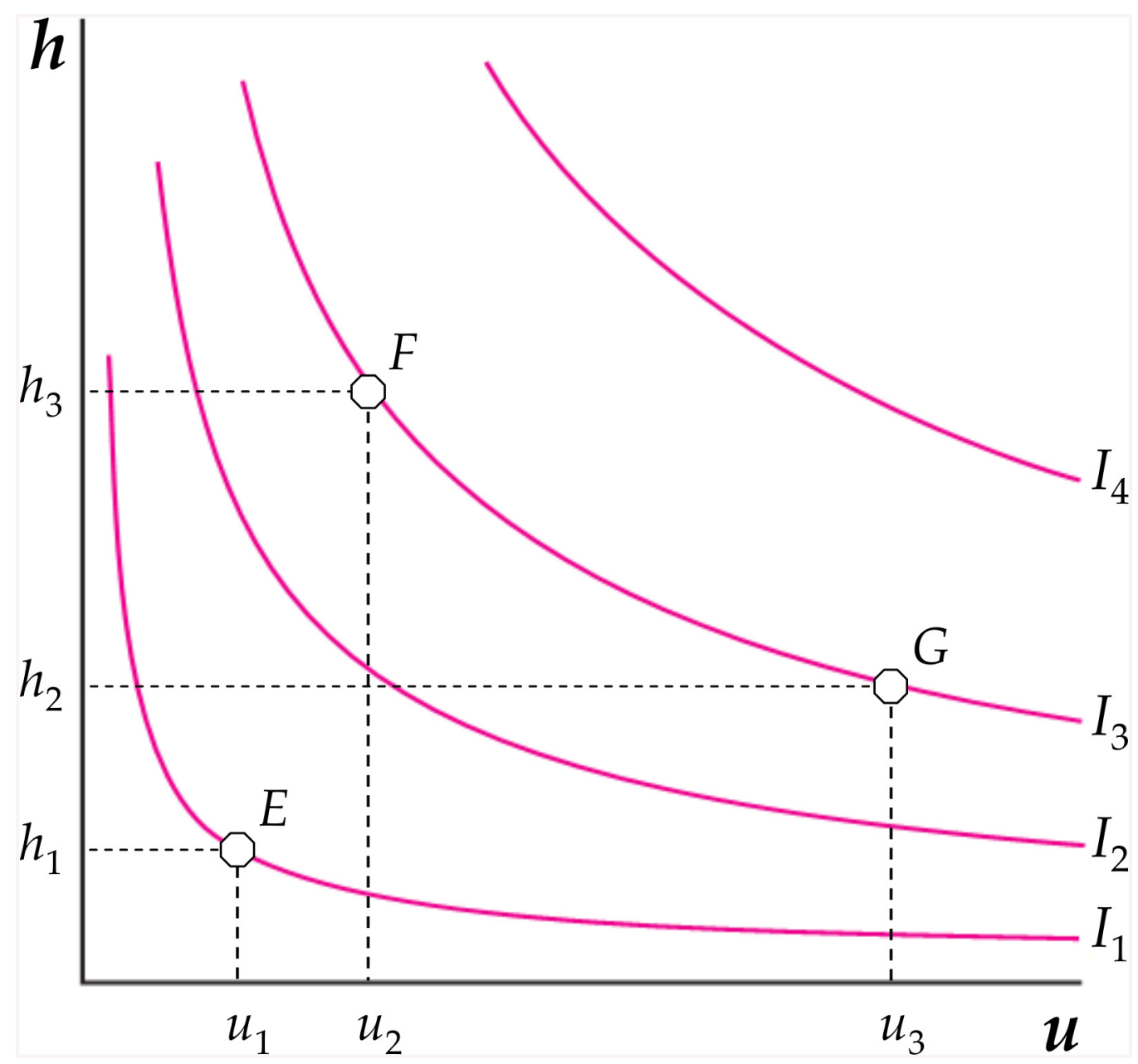

Fig 3. Indifference curves for $u$ and $h$.

doi:10.1371/journal.pone.0141834.g003

In this simplified model, people's exposure to NCD lifestyle risk factors depends on consumption choices of $h$ and $u$. These kinds of choices, in turn, depend not only on preferences, but also on budget constraints, i.e. on average income $(Y)$ and goods prices $\left(p_{h}\right.$ and $\left.p_{u}\right)$. This is shown in Fig 4 , where each straight line $\left(B_{2}, B_{3}\right.$ and $\left.B_{4}\right)$ indicates which consumption bundles are affordable at different income levels. If $Y=Y_{2}$, for example, $B_{2}$ shows all feasible quantities of $h$ and $u$ for which - given $p_{h}$ and $p_{u}$-total expenditure equals a consumer's income (i.e., $p_{h} h$ $+p_{u} u=Y_{2}$ or, rearranging, $\left.h=\left(Y_{2} / p_{h}\right)-\left(p_{u} / p_{h}\right) \times u\right)$.

With a limited purchasing power, the consumer's optimal bundle is the result of a constrained optimisation problem: choosing the combination of $h$ and $u$ that yields the highest utility, given the restrictions imposed by income and prices. For a given constraint, such as $B_{2}$ in Fig 4, the optimal bundle $\left(h_{2}, u_{2}\right)$-and thus, the more or less risk prone lifestyle-occurs at the tangency between the budget line and the highest (i.e., further from the origin) indifference curve (point $R$ ).

The diminishing value of $\eta_{U Y}$, reported in Table 4, finds a straightforward explanation in Fig 4. Consumption patterns are characterised by a hierarchical structure, determined by the interplay between biological, economic and social factors. Therefore, the demand for goods, such as $h$ and $u$, does not expand proportionally with economic growth and human 


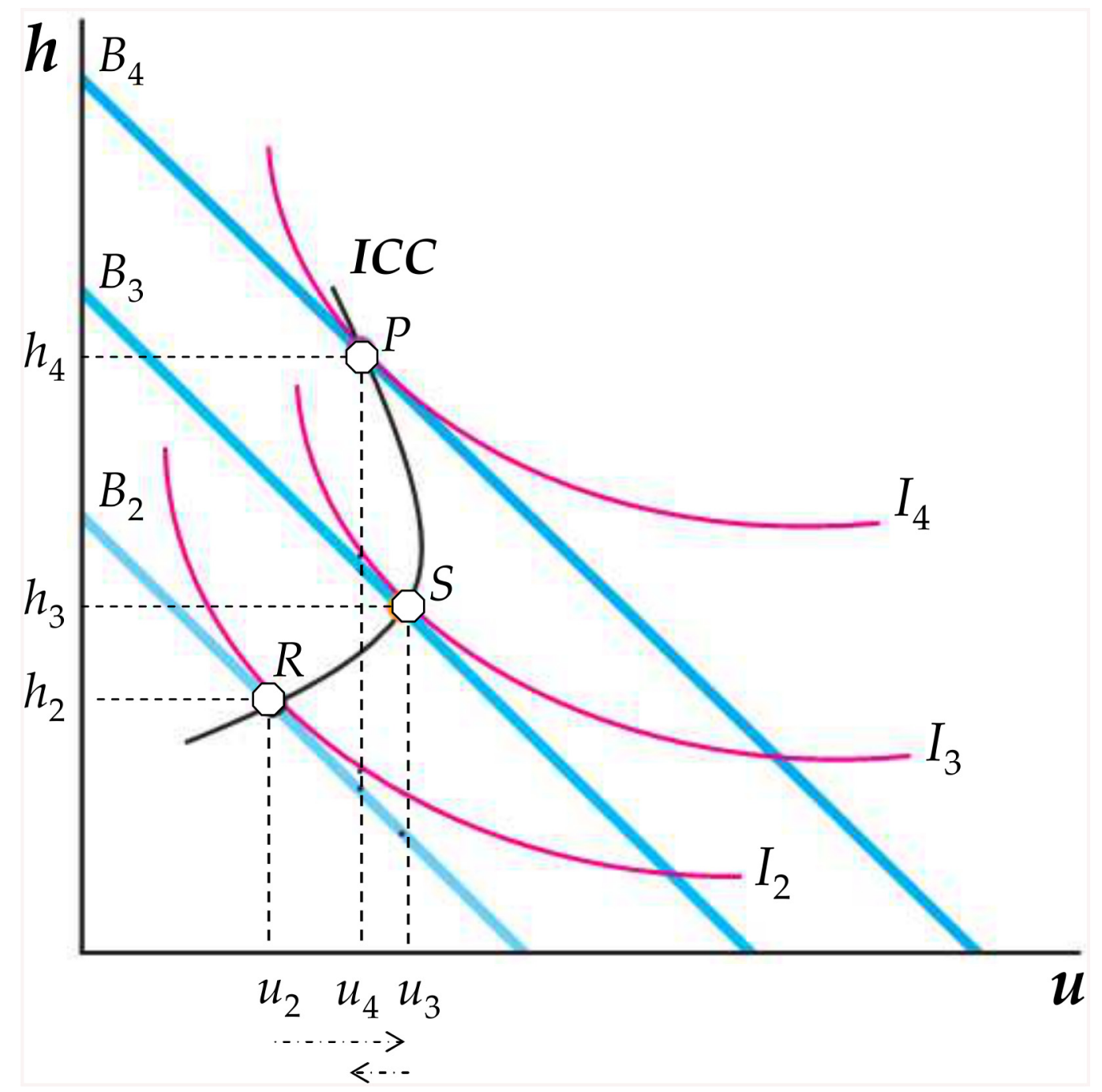

Fig 4. The income consumption curve for $u$ and $h$.

doi:10.1371/journal.pone.0141834.g004

development. Above threshold levels of disposable income (such as $Y_{3}$ or $Y_{4}$, in Fig 4), people start following new lifestyles, modifying accordingly their consumption patterns [29,30]. Specifically, as income increases (for example, from $Y_{2}$ to $Y_{4}$ ), the budget constraint shifts outward (from $B_{2}$ to $B_{4}$ ). Consumers can reach indifference curves further from the origin and choose better (i.e., higher utility) consumption bundles such as $\left(h_{3}, u_{3}\right)$ or $\left(h_{4}, u_{4}\right)$.

The path connecting the utility-maximising bundles (points $R, S, P$ in Fig 4 ) shows how consumers change their health-related behaviours as income increases, ceteris paribus. This income-consumption curve (ICC) becomes steeper at higher levels of utility, showing that a decreasing proportion of income is spent on $u$ (the demand for $u$ increases, but less than income). According to our results, this is the case in low, middle and upper-middle income countries, where $\eta_{U Y}$ is less than one, and tends to diminish as income rises. Conversely, the backward bending portion of ICC above point $S$ indicates a shifting toward more healthy lifestyles. This case applies to high income countries, where $\eta_{\mathrm{UY}}$ is negative; hence, $u$ become an inferior good (that is, a good for which consumption decreases when income rises). 
These findings may have several interesting implications for public health. The income elasticity of health outcomes $\left(\eta_{H Y}\right)$ is a frequently used metric to summarise the impact of economic growth on people's health [31]. Specifically, $\eta_{H Y}$ is the percentage change in a given measure of a population's health status $(H)$ divided by the percentage change in income per capita $(Y)$. That is, $(\Delta H / H) /(\Delta Y / Y)$, or more usefully:

$$
\eta_{H Y}=\frac{d H}{d Y} \times \frac{Y}{H}
$$

which gives the percentage change in health outcomes resulting from a $1 \%$ change in average income. Now, let us denote with $H$ the age-standardised incidence rate of the leading NCDs. In any population, given the inborn characteristics of individuals $(Z), H$ depends on at least two fundamental factors: health care $(H C)$ and health-related behaviours $(H B)$ [32]. This relationship between health inputs and outcome may be described by a health production function' [33] as follows:

$$
H=f(H C, H B, Z)
$$

where $H C$ is a measure of the resources devoted to preventing NCDs, and $H B$ is a summary measure of people's exposure to NCD lifestyle risk factors.

In this paper, we focus on $H B$. For our purposes, both $Z$ and $H C$ can be treated as exogenous variables of the model, whose value is taken as given. Thus, we can rewrite Eq 11 as a relation between incidence rate and health-related behaviours-i.e. $H=f(H B)$ - holding all other factors affecting $H$ constant. Moreover, we are able to replace the generic $H B$ variable with the $U B I$, and make the dependence of health-related lifestyles from income per capita explicit: $U B I$ $=g(Y)$. As a result, Eq 11 becomes:

$$
H=f[g(Y)]
$$

This expression simply states that changes in income lead to changes in health-related habits; in turn, changes in health-related habits affect a population's exposure to lifestyle risk factors, and thus, lead to changes in health outcomes.

Finally, in order to measure the magnitudes of these changes [34], let us differentiate $H$ with respect to $Y$ in Eq 12. By applying the chain rule, this gives:

$$
\frac{d H}{d Y}=\frac{d H}{d U B I} \times \frac{d U B I}{d Y}
$$

Remembering (from Eq 10) that $\eta_{H Y}$ is $d H / d Y$ multiplied by the income-health ratio $(Y / H)$, and since $Y / H$ can also be written as $(Y / U B I) \times(U B I / Y)$, after some manipulations, the expression for $\eta_{H Y}$ becomes:

$$
\eta_{H Y}=\frac{d H}{d U B I} \frac{U B I}{H} \times \frac{d U B I}{d Y} \frac{Y}{U B I}
$$

In Eq 14, the 'reactivity' of the NCD incidence rate to economic growth results from the product of two key factors: the elasticity of a population's health status with respect to healthrelated lifestyles $\left(\eta_{H U}\right)$ multiplied by the elasticity of a population's health-related lifestyles with respect to income per capita (that is, by the income elasticity of the $U B I, \eta_{U Y}$ ):

$$
\eta_{H Y}=\eta_{H U} \times \eta_{U Y}
$$

It is worth noting that $\eta_{H U}$ and $\eta_{U Y}$ are output and income elasticity, respectively. On the one hand, $\eta_{H U}$ tells us how much $H$ responds to a $1 \%$ change in the UBI. Its value is, therefore, 
largely determined by the technical and biological constraints that characterise the health production process. On the other hand, $\eta_{U Y}$ tells us how much the $U B I$ responds to a $1 \%$ change in $Y$. Its value depends mainly on people's preferences as consumers and citizens about healthrelated lifestyle choices; thus, it could be properly manipulated by public health prevention programmes.

As a result, to understand and predict, at a macroeconomic level, how $H$ may evolve in a growing economy_-given $Z$ and $H C$-it is crucial to measure not only $\eta_{H U}$, but also $\eta_{U Y}$, which gives both the direction and magnitude of a population's changes in health-related lifestyles in response to better living standards. In light of Eq 15, our results suggest that, other things being equal, up to a per capita income of about $\$ 40,500$ (i.e., an extremely high income level), economic growth tends to increase unhealthy habits. Therefore, albeit decreasing and less than one, the positive value of $\eta_{U Y}$ for a large majority of countries strongly confirms the role of primordial and primary prevention to reduce the incidence of NCDs throughout the world.

\section{Conclusions}

The main purpose of this study was to describe a straightforward method for calculating, at a macroeconomic level, an index of NCD-related unhealthy behaviours. Despite its highly simplified nature, the UBI creates a meaningful country ranking and offers some insights into the relation between economic development and the incidence rate of the main NCDs. Our results suggest that a significant part of the world's population still lives in regions where economic growth tends to push people toward 'Westernised', unhealthy behaviours, and, thus, supports the need for worldwide effective policy action to control lifestyle-related risk factors for NCDs.

Further research, however, needs to be done in order to develop a more comprehensive measure. The UBI suffers from a number of important limitations; it should be considered a first attempt in this research line. Specifically, although data on alcohol consumption include both recorded and unrecorded (i.e., homemade alcohol, illegally produced or sold outside normal government controls) alcohol [17], we do not distinguish between different alcoholic beverages or between moderate consumption and heavy episodic drinking. Similarly, data on cigarette consumption record manufactured and non-manufactured tobacco [18], but we do not consider that the total exposure to tobacco health risks is related to both intensity and prevalence of smoking. Furthermore, a correct measure of eating habits, as noted in the Appendix, has to be based on more dietary characteristics and different nutrient intake besides calorie, total fat and protein consumption. Finally, and perhaps more crucially, working with average country data fails to capture the key role of income inequality in health-related lifestyles within populations.

\section{Supporting Information}

S1 Appendix. The nutrition dimension index (NUTX), containing Fig A ECI and obesity prevalence, and Fig B. NBD and obesity prevalence. (DOC)

S1 File. Data and results, containing Tables A, B, C, D and E. Table A, Source and short description of each variable. Table B, Raw data (full database, with 159 countries). Table C, Indicators and indices (reduced database, with 112 countries). Table D, Country ranking. Table E, ECI, NBD and obesity.

(XLS) 


\section{Acknowledgments}

The author would like to thank the anonymous reviewers for their helpful and constructive comments that greatly improved the final version of the paper. All remaining errors are my own.

\section{Author Contributions}

Conceived and designed the experiments: FF. Performed the experiments: FF. Analyzed the data: FF. Wrote the paper: FF.

\section{References}

1. Bloom DE, Cafero ET, Jané-Llopis E, Abrahams-Gessel S, Bloom LR, Fathima S, et al. The global economic burden of non-communicable diseases. Geneva: World Economic Forum and Harvard School of Public Health; 2011.

2. WHO. Global status report on non-communicable diseases. Geneva: World Health Organization; 2014.

3. Suhrcke M, Nugent RA, Stuckler D, Lorenzo R. Chronic disease: An economic perspective. London: Oxford Health Alliance; 2006.

4. WHO. Globalisation, diets, and NCDs. Geneva: World Health Organization; 2002.

5. WHO. Global strategy on diet, physical activity and health. Geneva: World Health Organization; 2004.

6. NCD Alliance. Annual report 2014-2015. Geneva: Non-Communicable Diseases Alliance; 2015.

7. WHO. Preventing chronic diseases: A vital investment. Geneva: World Health Organization; 2005.

8. Bonita R, Beaglehole R, Kjellström T. Basic epidemiology. 2nd ed. Geneva: World Health Organization; 2006.

9. Ezzati M, Riboli E. Can non-communicable diseases be prevented? Lessons from studies of populations and individuals. Science. 2012; 337(6101): 1482-1487.

10. Kontis V, Mathers CD, Rehm J, Stevens GA, Shield KD, Bonita R, et al. Contribution of six risk factors to achieving the $25 \times 25$ non-communicable disease mortality reduction target: A modelling study. The Lancet. 2014; 384(9941): 427-437. doi: 10.1016/S0140-6736(14)60616-4

11. Loef $\mathrm{M}$, Walach $\mathrm{H}$. The combined effects of healthy lifestyle behaviours on all cause mortality: A systematic review and meta-analysis. Preventive Medicine. 2012; 55(3): 163-170. doi: 10.1016/j.ypmed. 2012.06.017 PMID: 22735042

12. Ding $D$, Do A, Schmidt H-M, Bauman AE. A widening gap? Changes in multiple lifestyle risk behaviours by socioeconomic status in New South Wales, Australia, 2002-2012. PLoS ONE. 2015; 10(8): e0135338. doi: 10.1371/journal.pone.0135338 PMID: 26291457

13. UNDP. Human development report $2013-T e c h n i c a l$ notes. New York: United Nations Development Programme; 2013.

14. OECD. Handbook on constructing composite indicators. Paris: Organisation for Economic Co-operation and Development; 2005.

15. WHO. Global health risk. Mortality and burden of disease attributable to selected major risks. Geneva: World Health Organization; 2009.

16. Klugman J, Rodríguez F, Choi H-J. The HDI 2010: New controversies, old critiques. Human Development Reports; 1 April 2011.

17. WHO. Global status report on alcohol and health, 2014. Geneva: World Health Organization; 2014.

18. Ng M, Freeman MK, Fleming TD, Robinson M, Dwyer-Lindgren $L$, Thomson B, et al. Smoking prevalence and cigarette consumption in 187 countries, 1980-2012. JAMA. 2014; 311(2): 183-192. doi: 10. 1001/jama.2013.284692 PMID: 24399557

19. FAO. Food and nutrition in numbers, 2014. Rome: Food and Agriculture Organization of the United Nations; 2014.

20. FAO, WHO. Diet, nutrition and the prevention of chronic diseases. Geneva: World Health Organization; 2003.

21. Ronksley PE, Brien SE, Turner BJ, Kenneth J Mukamal KJ, Ghaly WA. Association of alcohol consumption with selected cardiovascular disease outcomes: A systematic review and meta-analysis. British Medical Journal. 2011; 342(22): 671-671. doi: 10.1136/bmj.d671 
22. WCRF/AICR World Cancer Research Fund and American Institute for Cancer Research. Food, nutrition, physical activity, and the prevention of cancer: A global perspective. Washington, DC: AICR; 2007.

23. Schutze M, Boeing H, Pischon T, Rehm J, Kehoe T, Gmel G, et al. Alcohol attributable burden of incidence of cancer in eight European countries based on results from prospective cohort study. British Medical Journal. 2011; 342(7); 1584-d1584. doi: 10.1136/bmj.d1584

24. Nichols $\mathrm{M}$, Scarborough $\mathrm{P}$, Allender $\mathrm{S}$, Rayner $\mathrm{M}$. What is the optimal level of population alcohol consumption for chronic disease prevention in England? Modelling the impact of changes in average consumption levels. British Medical Journal. 2012; 2(3): 957. doi: 10.1136/bmjopen-2012-000957

25. Arranz S, Chiva-Blanch G, Valderas-Martínez P, Medina-Remón A, Lamuela-Raventós RM, Estruch R. Wine, beer, alcohol and polyphenols on cardiovascular disease and cancer. Nutrients. 2012; 4(7): 759-781. doi: 10.3390/nu4070759 PMID: 22852062

26. UNDP. Human development report, 2014. New York: United Nations Development Programme; 2014.

27. Auld MC, Powell LM. Economics of food energy density and adolescent body weight. Economica. 2009; (76: ): 719-740. doi: 10.1111/j.1468-0335.2008.00709.x

28. Forster M. The meaning of death: Some simulations of a model of healthy and unhealthy consumption. Journal of Health Economics. 2001; 20(4): 613-638. doi: 10.1016/s0167-6296(01)00080-7 PMID: 11463191

29. Argyrous $\mathrm{G}$. Endogenous demand in the theory of transformational growth. In: Setterfield M, editor. The economics of demand-led growth. New York: Edward Elgar; 2002.

30. Kearney J. Food consumption trends and drivers. Philosophical Transactions of the Royal Society B: Biological Sciences. 2010; 365(1554): 2793-2807. doi: 10.1098/rstb.2010.0149

31. Bishai $D, O$ 'Neil J. Economic growth and better health: The UK's surprising progress. The Lancet. 2012; 380: 649. doi: 10.1016/S0140-6736(12)61370-1

32. Folland S, Goodman AC, Stano M. The economics of health and health care. 7th ed. New York: Prentice Hall; 2012.

33. Sloan FA, Hsieh CR. Health economics. Cambridge: MIT Press; 2012.

34. Cohen IK, Ferretti F, McIntosh B. A simple framework for analysing the impact of economic growth on non-communicable diseases. Cogent Economics \& Finance. 2015; 3: 1045215. doi: 10.1080/ 23322039.2015.1045215 